\title{
Evaluación de los Pretratamientos de Deshidratación Osmótica y Microondas en la Obtención de Hojuelas de Mango (Tommy Atkins)
}

\author{
Maribel García-Paternina ${ }^{(1)}$, Armando Alvis-Bermudez ${ }^{(1)^{*}}$, Carlos A García-Mogollon ${ }^{(2)}$ \\ (1) Facultad de Ingenierías, Universidad de Córdoba, Programa de Ingeniería de Alimentos, \\ Grupo de Investigación Procesos y Agroindustria de Vegetales, Carrera 6 № 76-103, Km 3, vía Cereté. \\ Córdoba - Colombia (e-mail: aalvis2@hotmail.com). \\ (2) Facultad de Ingeniería, Universidad de Sucre. Sincelejo, Sucre, Colombia.
}

${ }^{*}$ Autor a quien debe ser dirigida la correspondencia.

Recibido Ene. 29, 2015; Aceptado Abr. 2, 2015; Versión final May. 8, 2015, Publicado Oct. 2015

\section{Resumen}

En el presente estudio, se evaluó el efecto de distintos procesos de secado de Mango (Tommy Atkins). Se usó deshidratación osmótica $\left(65^{\circ}\right.$ Brix de 37 a $40^{\circ} \mathrm{C}$ por $60 \mathrm{~min}$ ), microondas (560 W por $7 \mathrm{~min}$ ) y secado combinado $\left(70{ }^{\circ} \mathrm{C}\right.$ en una estufa tipo bandeja y al sol. Mangos con 11 a $14^{\circ}$ Brix y humedad $80 \%$ fueron dimensionados a $1 \times 1 \times 0.4 \mathrm{~cm}$. La pérdida de peso y el tiempo de secado en los mangos pre tratados presentaron diferencias significativas $(p \leq 0.05)$. La mayor pérdida de peso fue de $66.0 \%$ en el pretratamiento combinado. Los parámetros L (Luminosidad) y $b^{*}$ (azul amarillo) disminuyeron $(p \leq 0.05)$. El pretratamiento combinado es el que más eliminó agua y redujo los tiempos de secado. Sin embargo, el pre tratamiento de deshidratación osmótica fue el que mejor mantuvo las características de apariencia de las hojuelas de mango deshidratadas.

\section{Evaluation of the Osmotic Dehydration Pretreatment and Microwave in the Obtaining of Flakes of Mango (Tommy Atkins)}

\begin{abstract}
In the present study the effect of different drying processes of Mangos (Tommy Atkins) is studied. Osmotic dehydration ( $65^{\circ}$ Brix of 37 to $40^{\circ} \mathrm{C}$ for $60 \mathrm{~min}$ ), microwave drying (560 W by $7 \mathrm{~min}$ ) and combined as pretreatment of drying $\left(70{ }^{\circ} \mathrm{C}\right.$ in an oven tray type and under the sun) were considered. Mangos with 11 to $14^{\circ}$ Brix and humidity $80 \%$ were rated cut to dimensions $1 \times 1 \times 0.4 \mathrm{~cm}$. The weight loss and the drying time in the pretreated showed significant differences $(p \leq 0.05)$; the greatest weight loss was $66.0 \%$ in the pretreatment combined. The parameters $L$ (luminosity) and $b^{*}$ (blue-yellow) decreased $(p \leq 0.05)$. The combined pre-treatment is the one that eliminated more water and reduced drying times. However, osmotic dehydration was the one that best maintained the appearance features of dried mango flakes
\end{abstract}

Keywords: mango, flakes, osmotic dehydration, microwave, complementary drying 


\section{INTRODUCCIÓN}

La deshidratación o desecación de alimentos consiste en eliminar la mayor cantidad posible de agua o humedad del alimento seleccionado bajo una serie de condiciones controladas como temperatura, humedad, velocidad y circulación del aire. El agua es el elemento básico para la vida humana, pero también para la vida microbiana, por lo que, al retirarla, ayuda a darle una vida útil y más prolongada al alimento, el desecado provoca que el alimento en cuestión se reduzca en tamaño debido a que ha perdido gran parte de su volumen (agua), y como resultado se obtiene un alimento de consistencia más liviana y pequeña de un buen sabor y olor el cual es muy resistente y de fácil transportación. Todos estos estudios están dirigidos a mejorar el proceso y la calidad de hojuela, con un riesgo mínimo de descomposición o crecimiento microbiano (Fito, 1994). El proceso de deshidratación disminuye la actividad acuosa (Aw) la cual es una medida de la disponibilidad del agua para las reacciones químicas y bioquímicas y para el desarrollo de microorganismos. Existen varios procesos de deshidratación que tienen el inconveniente de someter el producto a altas temperaturas que alteran las propiedades organolépticas y nutricionales, usando técnicas apropiadas se puede obtener mango con cualidades físico-químicas y sensoriales similares al producto fresco (Moreno et al., 2010). Una alternativa es la eliminación de humedad previa al secado aplicando la deshidratación osmótica (DO) o la tecnología emergente por microondas (MW); la primera es un método no térmico de deshidratación mediante el cual se logra obtener productos de humedad intermedia con muy buena calidad organoléptica (Reppa, 2011). Zuluaga et al. (2010) estudiaron la combinación de tecnologías aplicando DO como pretratamiento al secado convencional donde evaluaron la influencia del proceso y la temperatura de secado (60 y $70^{\circ} \mathrm{C}$ ) sobre las características físicas del mango (Tommy Atkins), Ochoa et al. (2012) evaluaron las características de secado en ventana refractaria (RW) con un baño de agua a una temperatura de $92{ }^{\circ} \mathrm{C}$ de rebanadas de mango (1 y $2 \mathrm{~mm}$ de espesor), Villalpando et al. (2011) aplicaron microondas (1000 W) de tres diferentes rebanadas de mango (ovalado, longitudinal y transversal) como complemento al secado de muestras osmodeshidratadas las deshidratadas de mango, el tiempo de tratamiento, el efecto sobre la perdida de agua, vitamina $\mathrm{C}$ y cambios de color.

La pérdida de peso, tiempo de secado, cambios de color y preferencia de los consumidores son factores afectados por la combinación de deshidratación osmótica, microondas y combinación (DO y MW) previa al secado en estufa y solar. Para una buena conservación de la materia prima y del producto final en hojuelas de mango de la variedad Tommy Atkins, se evaluó la influencia de los pre-tratamientos sobre el proceso de secado y las características físicas del mango. En la conservación de frutas, el secado combinado con pretratamientos juega un papel importante en el desarrollo de nuevos productos, la DO como pre-tratamiento, favorece la disminución de los cambios adversos que ocurren durante el uso de métodos combinados, mientras que el secado ayuda a conservar por más tiempo la fruta (Zuluaga et al., 2010). El objetivo de esta investigación fue evaluar el efecto del pretratamiento aplicado por microondas y deshidratación osmótica sobre las características de la hojuela de mango (Tommy Atkins) deshidratada por secado convencional, que al final se verá reflejado en los tiempos de secado y las características de la hojuela de mango deshidratada.

\section{MATERIALES Y MÉTODOS}

\section{Obtención y adecuación de la materia prima}

Los mangos (Tommy Atkins) utilizados como materia prima se seleccionaron con un índice de madurez de 0.125 , de 11 a $14{ }^{\circ}$ Brix y humedad $80 \pm 1 \%$ los cuales se sometieron a operaciones de lavado, pelado, troceado para obtener las hojuelas de una dimensión de $1 \mathrm{~cm} \times 1 \mathrm{~cm} \times 0.4 \mathrm{~cm}$. Los sólidos solubles se determinaron por refractometría (AOAC 932.12/90). La acidez se midió por el método de titulación potenciométrica (método AOAC 942.05/90). El índice de madurez se calculó empleando la relación de sólidos solubles/ acidez.

\section{Pretratamiento al secado}

Deshidratación osmótica (DO): Para el tratamiento osmótico se utilizó una solución de sacarosa de $65^{\circ}$ Brix por 60 min y temperatura ambiente de 37 a $40^{\circ} \mathrm{C}$. Las hojuelas de mango (100 g) se dispusieron en bolsas plásticas para posteriormente sumergirlas en soluciones osmóticas con relación a la masa de muestra: volumen solución 1:5. Una vez culminado el tiempo del tratamiento, la muestra deshidratada se escurrió por 15 min en un colador para eliminar el exceso de solución osmótica.

Deshidratación por microondas (MW): Se utilizó un horno microondas doméstico de marca Haceb HM-1.1 con una frecuencia de $2450 \mathrm{MHz}$ y dimensiones internas de $354 \mathrm{~mm}$ x $228 \mathrm{~mm} \times 373 \mathrm{~mm}$. Se tomaron $50 \mathrm{~g}$ de muestra y se distribuyeron uniformemente en el plato del microondas a una potencia de $560 \mathrm{~W}$ por $7 \mathrm{~min}$. La 
masa de las muestras durante el secado se obtuvieron pesando en una balanza analítica (MettlerPS4000 con exactitud $\pm 0.01 \mathrm{~g}$ ).

Combinado: Se aplicó el pretratamiento de deshidratación osmótica seguido de microondas, acorde a los procedimientos preliminares.

\section{Tratamiento de secado}

Secado al sol: Se utilizaron bandejas de $50 \mathrm{~g}$ de muestra y se expusieron a la radiación solar de 9:00 a las 15:00 hasta masa constante, en la sede Berastegui ubicada en el corregimiento de Berastegui, municipio de Ciénaga de Oro, Departamento de Córdoba, Colombia.

Secado con convección: Se usó un horno convección marca Technicook modelo MINICONV con dimensiones $580 \mathrm{~mm}$ de ancho, $630 \mathrm{~mm}$ de profundidad, $435 \mathrm{~mm}$ de alto, tipo bandeja del Laboratorio de GIPAVE en la sede Berastegui ubicada en el corregimiento de Berastegui, municipio de Ciénaga de Oro, Departamento de Córdoba, Colombia. La temperatura de secado fue de $70^{\circ} \mathrm{C}$.

\section{Pérdida de peso}

Se realizaron registros de la pérdida de peso $(\mathrm{g})$ de las hojuelas al inicio $\left(\mathrm{P}_{\mathrm{i}}\right)$ y al final $\left(\mathrm{P}_{\mathrm{f}}\right)$ de cada pretratamiento. Se expresaron como porcentaje de pérdida de peso (\%PP) a partir de la ecuación:

$$
\% P P=\frac{P_{i}-P_{f}}{P_{i}} \times 100
$$

Las curvas de secado se realizaron con las la pérdida de peso de la muestra cada 10 min en las primeras 2 h de proceso y luego cada 30 min hasta peso constante. Los pesos obtenidos se convierten en términos de humedad libre empleando la ecuación:

$$
\mathrm{X}_{\mathrm{t}}=\frac{\mathrm{W}-\mathrm{W}_{\mathrm{s}}}{\mathrm{W}_{\mathrm{s}}}
$$

Dónde: $\mathrm{W}$ es el peso del sólido húmedo ( $\mathrm{kg}$ totales de agua más sólido seco), Ws es el peso del sólido seco ( $\mathrm{kg}$ sólido seco), y $\mathrm{X}_{\mathrm{t}}$ es la relación entre el peso del agua y el peso del sólido seco (MS) a un determinado tiempo ( $\mathrm{kg}$ de agua/kg MS).

\section{Color}

El análisis de color se realizó determinando las coordenadas de color del espacio CIELAB ( $L^{*}$ : luminosidad o claridad; $a^{*}$ : coordenada rojo-verde y $b^{*}$ : coordenada amarillo-azul). Las mediciones se hicieron con un colorímetro Colorflex EZ 45 (HunterLab®). El colorímetro se calibró con un plato de cerámica estándar de color verde y blanco estándar antes de su lectura.

\section{Diseño experimental}

Se aplicó un diseño experimental completamente al azar con arreglo factorial $4 \times 2$ correspondiente a la variable categórica pretratamiento con cuatro niveles (DO: deshidratación osmótica; MW: microondas, SinP: sin pretratamiento y DOMW: combinado) y el tratamiento de secado al sol y convectivo (E); 3 repeticiones para un total de 24 unidades experimentales. Para comparar las parámetros CIELab* de los tratamientos con el mango fresco se aplicó la prueba de comparación de Dunnett. Los datos fueron analizados por el programa STATISTICA (versión 7, 2004, StatSoft. Inc., Tulsa, EE.UU).

\section{RESULTADOS Y DISCUSIÓN}

\section{Caracterización físico-química de las hojuelas frescas de mango}

La evaluación de las características físico-químicas de los frutos de mango permitió establecer el estado de madurez para la aplicación de la deshidratación osmótica y por microondas para la posterior operación de secado en estufa y sol. Las propiedades físico-químicas del mango usado fueron $12.3 \pm 0.7^{\circ} \mathrm{Brix}$ y $0.69 \pm 0.1 \%$ acidez expresada como ácido cítrico, correspondiendo a un grado 4 de madurez teniendo en cuanta la escala establecida para el mango variedad Tommy Atkins. 
Pretratamientos en las hojuelas de mango

La pérdida de agua de las hojuelas de mango presento diferencias significativas $(p \leq 0.05)$ entre los pre tratamientos (Figura 1) de deshidratación osmótica (DO), microondas (MW), el combinado (DOMW) y sin pretratamiento (SinP). El tratamiento que permitió la mayor eliminación de agua fue el combinado con un $66.0 \%$ y el menor fue a las condiciones de $560 \mathrm{~W}$ y $7 \mathrm{~min}$ a los que se sometieron con el MW.

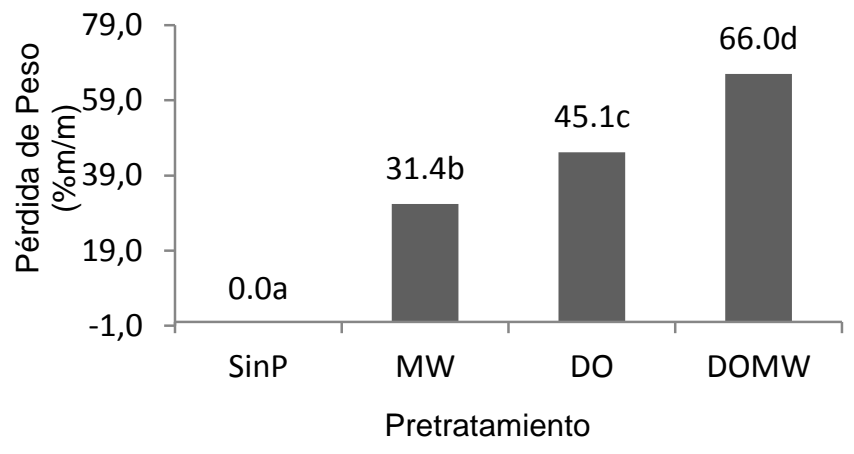

Fig. 1: Pérdida de peso del mango en los pretratamientos

Lo anterior muestra el efecto que tiene cada pretratamiento sobre la pérdida de agua de las hojuelas de mango y lo reportado por otros autores como se muestra en la tabla 1, indicando que la inmersión de la fruta en una solución hipertónica involucra procesos en los cuales tienen lugar la pérdida de agua y la ganancia de soluto a través de la membrana del material biológico (Wang et al., 2010). Estos fenómenos de transferencia de masa son afectados por factores como la presión, la temperatura, el soluto y la concentración de la solución, la relación jarabe: fruta, el grado de agitación del medio, entre otros. EI MW es una tecnología no convencional de calentamiento, cuyo mecanismo implica el paso de ondas a través de la estructura de la hojuela de mango calentando desde el centro generando un perfil de temperatura de mayor a menor del centro hacia fuera, logrando que el agua se caliente y migre hacia la superficie de la hojuela hasta que alcance la temperatura de evaporación. Esto aumenta los canales para movimiento y salida de agua hacia las capas superficiales, lo que favorece la deshidratación en el tratamiento de secado.

Tabla 1: Pérdida de peso (PP) en deshidratación osmótica

\begin{tabular}{|l|c|c|c|c|c|l|}
\hline Fruta & $T\left({ }^{\circ} \mathrm{C}\right)$ & ${ }^{\circ}$ Brix & Tiempo $(\mathrm{h})$ & Dimensiones $(\mathrm{cm})$ & $P P(\% \mathrm{~m} / \mathrm{m})$ & \multicolumn{1}{|c|}{ Referencia } \\
\hline Mango & Ambiente & 65 & 20 & $2.5 \times 2.5 \times 1$ & 76.13 & Calderón y Jurado (2008) \\
\hline Mango & 20 & 40 & 8 & $1.5 \pm 0.1$ & 17,06 & Zuluaga et al. (2010) \\
\hline Mango & $22-25$ & 67 & 12 & $11 \times 1.0 \times 2.5$ & 42,3 & Villalpando et al. (2011) \\
\hline Mango & 45 & 55 & 4 & $1 \times 1 \times 0.5$ & 46.68 & Zapata et al. (2012) \\
\hline Mango & 60 & 45 & 3 & $1 \times 1 \times 1$ & 75.00 & Mendoza y Muñoz, 2012 \\
\hline Mango & 30 & 65 & 1 & $2.6 \times 2.6 \times 0.5$ & 16.71 & Zou et al. (2013) \\
\hline Piña & 60 & 50 & 6 & $2 \times 5 \times 1$ & 64.32 & García et al. (2013) \\
\hline Mango & $37-40$ & 65 & 1 & $1 \times 1 \times 0.4$ & 45.11 & Este estudio \\
\hline
\end{tabular}

En consecuencia, la diferencia de mecanismos se refleja en las propiedades organolépticas de las hojuelas después del pretratamiento, evidenciándose que las osmodeshidratadas tienen mayor brillo y estructura que las tratadas por microondas debido a la ganancia de sólidos y el medio de solución azucarada en que se produce; además, las hojuelas tratadas con microondas se observan más aplanadas producto de que la estructura de la matriz de mango no es rígida y por lo tanto no compensa la salida del agua desde el interior. Cuando se combinaron los pre-tratamientos iniciando con la osmodeshidratación se redujeron los efectos negativos del microondas.

\section{Curva de pérdida de peso}

Al aplicar un pretratamiento los tiempos de secado al sol disminuyen pero siguen siendo mayores que los tiempos de secado en horno convectivo. La pérdida de peso en el secado para el pretratamiento con microondas evidencia el efecto que tiene en la velocidad de secado respecto al tratamiento de secado al sol 
y en horno convectivo (Figura 2). Para secar las muestras de mango al sol se necesitaron 510 min. cuando no se aplica el pretratamiento, mientras que para secar la misma muestra, que se le ha aplicado un pretratamiento por microondas, el tiempo disminuye a $380 \mathrm{~min}$. Se puede observar que la curva de secado del mango sin pretratamiento cuando son secadas en horno desciende más rápidamente (105 min) que las muestras con DO (150 min). Del mismo modo se observa cómo las curvas de DO secadas en horno descienden en forma más lenta, debido a la dificultad en la eliminación del agua por la formación de una corteza de la solución sacarifica del proceso de DO en la superficie de las hojuelas. Nieto et al. (2011), Mercer y Myhara (2009) y Zuluaga et al. (2010) obtuvieron resultados similares para mango deshidratado osmóticamente y secadas a temperaturas de $50-60^{\circ} \mathrm{C}$.
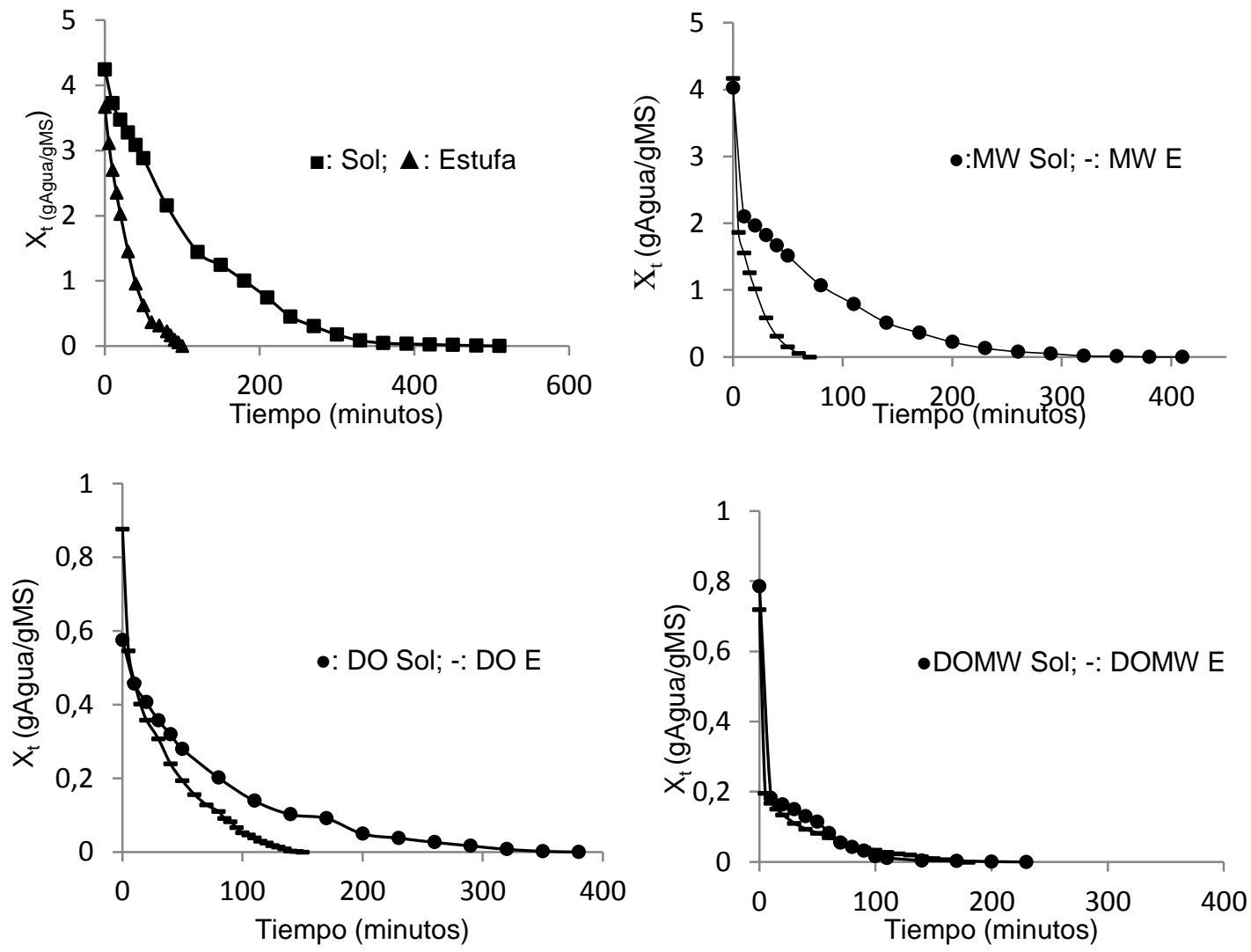

Fig. 2: Curvas de secado de las hojuelas de mango.

Los resultados obtenidos indican que la transferencia de masa asociada a la pérdida de agua en las hojuelas de mango fue más rápida con el método en estufa que en el secado al sol, dado que en el método en estufa se utilizan temperaturas mayores que en el secado al sol, generando mayor calor en la muestra, creando una mayor diferencia de presión de vapor entre el centro y la superficie debido al calentamiento. Se han llevado a cabo estudios similares sobre secado por aire caliente con pretratamiento osmótico en mango (Tabla 2) y otros productos como tomate (Telis et al., 2009), pimiento rojo (Vegas et al., 2009), remolacha (Vacarezza et al., 2010) fresas y cerezas (Lewicki y Lenart, 2011) y piña (Garcia et al., 2013).

Tabla 2: Pérdida de peso con pretratamiento de secado

\begin{tabular}{|l|c|c|c|c|c|l|}
\hline Fruta & $\begin{array}{c}\text { Pretratamient } \\
0\end{array}$ & Secado & $\begin{array}{c}T \\
\left({ }^{\circ} \mathrm{C}\right)\end{array}$ & Tiempo & $P P(\% \mathrm{~m} / \mathrm{m})$ & Referencia \\
\hline Mango & DO & Convectivo en bandeja & 70 & $8.0 \mathrm{~h}$ & 14.43 & Zuluaga et al. (2010) \\
\hline Mango & - & Convectivo en bandeja & 70 & $8.0 \mathrm{~h}$ & 10.2 & Zuluaga et al. (2010) \\
\hline Mango & MW & DO & $95-98$ & $180-182 \mathrm{~s}$ & $35-45$ & Villalpando et al.(2011) \\
\hline Mango & - & Liofilización & -20 & - & 77.02 & Sogi et al. (2014) \\
\hline Mango & DO & Convectivo en bandeja & 70 & $2.5 \mathrm{~h}$ & 45.1 & Este estudio \\
\hline Mango & MW & Convectivo en bandeja & 70 & $1.0 \mathrm{~h}$ & 31.4 & Este estudio \\
\hline
\end{tabular}

En la tabla 3 se muestran los tiempos para que las hojuelas de mango alcancen una humedad del 10\%. Del mismo modo se puede ver que al aplicar un pretratamiento de secado los tiempos disminuyen; por consiguiente se puede decir que para el método de secado al sol al aplicar un pretratamiento en microondas, 
deshidratación osmótica o una combinación los tiempos de secado se ven reducidos en un $37.3 \%, 57.8 \%$ y $81.7 \%$ respectivamente.

Tabla 3: Tiempos de secado hasta una humedad del $10 \%$ en mango

\begin{tabular}{|l|c|c|}
\hline Pretratamiento & \multicolumn{2}{|c|}{ Tiempo de secado (min) } \\
\hline & Sol & Estufa \\
\hline Sin Pretratamiento & $237.0 \pm 4.6 \mathrm{a}$ & $75.0 \pm 1.0 \mathrm{e}$ \\
\hline Microondas (MW) & $148.6 \pm 8.0 \mathrm{~b}$ & $28.6 \pm 1.2 \mathrm{f}$ \\
\hline Deshidratación Osmótica (DO) & $100.0 \pm 5.7 \mathrm{c}$ & $51.6 \pm 7.6 \mathrm{~g}$ \\
\hline Combinado (DOMW) & $43.3 \pm 5.7 \mathrm{~d}$ & $24 \pm 1.7 \mathrm{~h}$ \\
\hline
\end{tabular}

La DO retrasó el proceso de secado respecto a las otras muestras pretratadas, reduciendo solo un $31.2 \%$ el tiempo de secado en estufa, mientras que los otros pretratamientos redujeron un $61.9 \%$ (MW) y $68 \%$ (DOMW). Una explicación a esto se debe a que con la DO las hojuelas tienen una ganancia de solutos, la cual afecta el proceso de secado convencional dado a la cristalización de dichos solutos a nivel superficial. Resultados similares se obtuvieron al evaluar el efecto de deshidratación osmótica como pretratamiento al secado por aire caliente de cilantro y perejil (García et al., 2010), en mango (Zuluaga et al., 2010) y en papas combinando el secado convencional y por microondas (Della y Mascheroni, 2011).

\section{Color}

El efecto de los tratamientos sobre el color de las hojuelas de mango es observable a través del color amarrillo característico del mango maduro que presenta diferentes tonalidades que lo hacen más o menos atractivo. Los tratamientos sometidos a la DO en general adquieren un brillo y solidez en el color amarrillo, mientras que las tratadas al MW son más opacas. Lo cual indica que los pretratamientos influyeron en los cambios de color de las hojuelas.

Los cambios en los parámetros de color en diferentes estudios son debidos principalmente al índice de madurez, las condiciones de proceso y las características físicas; sin embargo, los parámetros del color más visiblemente afectados son $L$ y b al igual que en otros estudios realizados (Tabla 4). Con respecto a los parámetros CIELab* comparado con el mango fresco: el valor $L$ (Luminosidad) y b* (azul amarillo) presentaron diferencia significativa y el valor $\mathrm{a}^{*}$ (verde a rojo) no presentó diferencia significativa. De acuerdo a estos resultados, el valor de $b^{*}$ es menor en MW Sol (36.19 \pm 4.9$)$. En general, el mango fresco presenta un color amarillo intenso, pero al aplicar los tratamientos el parámetro $\left(b^{*}\right)$ y la $L$ disminuyen; mientras que $a^{*}$ no varió, indicando que no hay un efecto de quemado durante el proceso de secado. El tratamiento que más disminuyó las características de las hojuelas de mango respecto al mango fresco fue el pretratamiento de microondas y el secado solar (MW Sol).

Tabla 4: Valores de CIELab

\begin{tabular}{|c|c|c|c|c|c|c|}
\hline Fruta & Pretratamiento & $\mathrm{T}\left({ }^{\circ} \mathrm{C}\right)$ & L & $a^{*}$ & $b^{*}$ & Referencia \\
\hline Mango & $\mathrm{AC} / 24 \mathrm{~h}$ & 60 & 67.68 & 19.42 & 65.97 & Chen et al. (2007) \\
\hline Mango & $\operatorname{Sin} P$ & 70 & 64.97 & 15.38 & 54.27 & Zuluaga et al. 2010 \\
\hline Mango & DO & 70 & 57.09 & 16.19 & 41.09 & Zuluaga et al. 2010 \\
\hline Mango fresco & - & - & $74,19 \pm 1.1 \mathrm{a}$ & $11,45 \pm 0.1 \mathrm{a}$ & $57,02 \pm 0.2 a$ & Este estudio \\
\hline Mango & - & Sol & $60,80 \pm 2.6 b$ & $10,11 \pm 1.4 a$ & $39,59 \pm 2.8 b$ & Este estudio \\
\hline Mango & DO & 70 & $60,24 \pm 1.0 b$ & $11,66 \pm 0.8 a$ & $48,51 \pm 2.6 c$ & Este estudio \\
\hline Mango & DO & Sol & $58,57 \pm 2.3 b$ & $9,36 \pm 0.6 b$ & $44,37 \pm 0.8 b$ & Este estudio \\
\hline Mango & - & 70 & $56,80 \pm 3.0 \mathrm{~b}$ & $13,15 \pm 2.0 \mathrm{a}$ & $49,11 \pm 2.0 c$ & Este estudio \\
\hline Mango & MW & 70 & $56,07 \pm 3.1 b$ & $11,52 \pm 1.2 a$ & $47,82 \pm 1.0 \mathrm{c}$ & Este estudio \\
\hline Mango & DOMW & Sol & $55,39 \pm 3.8 b$ & $10,90 \pm 0.7 a$ & $45,49 \pm 2.7 b$ & Este estudio \\
\hline Mango & DOMW & 70 & $53,15 \pm 0.9 c$ & $12,13 \pm 0.2 a$ & $46,87 \pm 0.7 c$ & Este estudio \\
\hline Mango & MW & Sol & $50,96 \pm 1.1 d$ & $11,34 \pm 1.8 a$ & $36,19 \pm 4.9 d$ & Este estudio \\
\hline
\end{tabular}


El efecto del proceso de deshidratación en la superficie del mango se ve reflejado por los cambios y pérdida de color (Gómez, 2013). La oxidación extensiva provoca la pérdida en carotenoides y que es incrementada por el prolongado proceso de deshidratación (Zuluaga et al., 2010). La concentración de los agentes osmóticos es un factor que afecta el proceso (Phisut et al., 2013) y se han observado diferencias en el color y otros atributos sensoriales, comparando mango (Tommy Atkins) fresco e impregnado al vacío con sales de calcio a distintas concentraciones y mezclas (Ostos-A. et al., 2012). La deshidratación puede incrementar la concentración de carotenoides (Nimmanpipug y Therdthai, 2013; Phisut et al., 2013) causando un efecto contrario en el color del producto final.

\section{CONCLUSIONES}

Los pre-tratamientos de deshidratación osmótica, microondas y combinado extrajeron un alto porcentaje de humedad en las hojuelas reduciendo los tiempos de secado al sol y en estufa. Es el pretratamiento combinado el que más retiro agua y redujo los tiempos de secado; sin embargo, el pretramiento de deshidratación osmótica fue el que mejor mantuvo las características de apariencia de las hojuelas de mango deshidratadas.

Deshidratar las hojuelas de mango pretratadas reduce el tiempo de exposición a las condiciones térmicas de que contribuyen al detrimento de las características organolépticas de las hojuelas de mango.

El proceso de secado Microondas - Sol fue el de mayor decremento de los parámetros de color, traduciéndose en el tratamiento que más demerito las características de las hojuelas de mango respecto al mango fresco. Los tratamientos con deshidratación osmótica fueron los que mejor conservaron la apariencia de las hojuelas de mango.

\section{REFERENCIAS}

A.O.A.C.: Association of Official Analytical Chemist. Official Methods of Analysis, Section 954.10. 13th. Ed. Washington D.C., USA (1990)

Calderón, J. y J. Jurado, Conservation de babaco (carica pentágona), mango (magnifera índica) y pepino dulce (solanum muricatum) mediante deshidratación osmótica directa, Tesis de licenciatura, Universidad Técnica del Norte, Ecuador (2008)

Chen, J., C. Tai y B. Chen, Effects of different drying treatments on the stability of carotenoids in Taiwanese mango (Mangifera indica L.). Food Chemistry, 100: 1005-1010 (2007)

Della, P. y R. Mascheroni, Deshidratación de papas por el método combinado de secado: deshidratación osmótica, secado por microondas y convección por aire caliente. Revista Proyecciones, 9(2): 12-25 (2011)

Fito, P., Modelling of vacuum osmotic dehydration of food, Journal of Food Engineering, 22(1-4): 313-328 (1994)

García, M., J. Rusel, E. Rodriguez y E. Vargas, Aprovechamiento del cilantro (Coriandrumsativum) y perejil (Petrosilenumcrispum) aplicando procesos combinados de deshidratación. Universidad de Bogotá, Jorge Tadeo Lozano. Facultad de Ciencias Naturales e Ingenierías. Resultados de investigación, 3(2): 9-26 (2010)

García, A., S. Muñiz, A. Hernández, L. González, y D. Fernández, Análisis comparativo de la cinética de deshidratación Osmótica y por Flujo de Aire Caliente de la Piña (Ananas Comosus, variedad Cayena lisa). Revista Ciencias Técnicas Agropecuarias, 22(1): 62-69 (2013)

Gomez, R., Evaluación sensorial de láminas de mango (Manguifera indica L. cv. Keitt) fortificadas con cloruro de calcio mediante deshidratación osmótica con pulsos de vacío. Revista Venezolana de Ciencia y Tecnología de Alimentos, 4 (2): 157-169 (2013)

Lewicki, P. y A. Lenart, Effect of osmotic pre-treatment on convection drying of selected fruits, Memory I. Osmotic treatments for the food industry, Porto (2011)

Mendoza, R. y F. Muñoz, FTIR aplicada durante la deshidratación osmótica de mango Ataulfo (Magnífera indica L.). Superficies y Vacío, 25(1): 8-13 (2012)

Mercer, D. y R. Myhara, Improving the Operationof a Commercial Mango Dryer, Memory I. Using Food Science and Technology to Improve Nutrition and Promote National Development, Ontario, Canadá (2009) 
Moreno, A., D. León, G. Giraldo y E. Rios, Study of the physicochemical kinetics of mango (Mangifera indica L. Tommy Atkins) treated by combined methods of drying. Dyna 77 (162): 75-84 (2010)

Nieto, A., M. Castro y S. Alzadora, Kinetics of moisture transfer during air drying of blanched and/or osmotically dehydrated mango. Journal of Food Process Engineering 50(1): 175-185 (2011)

Nimmanpipug, N. y N. Therdthai, Effect of osmotic dehydration time on hot air drying and microwave vacuum drying of papaya. Food and Applied Bioscience Journal, 1(1): 1-10 (2013)

Ochoa, C., P. Quintero, A. Ayala y M. Ortiz, Drying characteristics of mango slices using the Refractance Window Technique. Journal of Food Engineering, 109: 69-75 (2012)

Ostos-A., S., A. Díaz y H. Suárez, Evaluación de diferentes condiciones de proceso en la fortificación de mango (Tommy Atkins) con calcio mediante impregnación a vacío. Revista Chilena de Nutrición, 39(2): 181 $190(2012)$

Phisut, N., M. Rattanawedee y K. Aekkasak, Effect of osmotic dehydration process on the physical, chemical and sensory properties of osmo-dried cantaloupe. International Food Research Journal, 20(1):189-196 (2013)

Reppa, A., J. Mandala, A. K. Kostaropoulos y G. D. Saravacos, Influence of solute temperature and concentration on the combined osmotic and air drying. Drying Technology, 17(7-8): 1449-1458 (2011)

Sogi, D., Muhammad Siddiq y Kirk D. Dolan, Total phenolics, carotenoids and antioxidant properties of Tommy Atkin mango cubes as affected by drying techniques, doi: 10.1016/j.Iwt.2014.04.015, LWT-Food Science and Technology (en línea) (2014)

Telis V., R. Murari R., J. Carrizo y J. Telis-Romero, Air -drying kinetics of osmotically pre-treated tomato, Memoria I. Nuevas fronteras en el siglo XXI, Valencia, España (2009)

Vaccarezza, L., J. Lombardi y J. Chirife, Kinetics of moisture movement during air drying of sugar beet root. Journal of Food Technology, 9(1): 317-327 (2010)

Vegas, A., L. Cortell, A. Andrés y P. Fito, Deshidratación osmótica como pretratamiento al secado por aire caliente de cubos de pimiento, Memoria I. nuevas fronteras en el siglo XXI, Valencia, España (2009)

Villalpando, J., E. Herrera, L. Amaya-Delgado, M. Godoy, J. Mateos y S. Rodríguez, Effect of Complementary Microwave Drying on Three Shapes of Mango Slices. Revista Mexicana de Ingeniería Química, 10(2): 281$290(2011)$

Wang, R., M. Zhang y A. S. Mujumdar, Effect of osmotic dehydration on microwave freeze-drying characteristics and quality of potato chips. Drying Technology, 28(6): 798-806 (2010)

Zapata, J. y A. Montoya, Deshidratación osmótica de láminas de mango cv. Tommy atkins aplicando metodología de superficies de respuesta. Universidad de Antioquia. Revista de Facultad de Ciencias Agrarias, 65(1): 6507-6518 (2012)

Zou, K., T. Jianwen, Li. Huang, Xinwei Dai y Baoyao Wei, Effect of osmotic pretreatment on quality of mango chips by explosion puffing drying. Food Science and Technology, 51: 253-259 (2013)

Zuluaga J., M. Cortes y E. Rodríguez, Evaluación de las características físicas de mango deshidratado aplicando secado por aire caliente y deshidratación osmótica. Universidad Nacional de Colombia, Medellín. Revista de la Facultad de Ingeniería, 25(4):127-135 (2010) 